\section{The clinical and laboratory evaluation of familial hemophagocytic lymphohistiocytosis and the importance of hepatic and spinal cord involvement: a single center experience}

Burcin Beken, ${ }^{1}$ Selin Aytac, ${ }^{2}$ Gunay Balta, ${ }^{2}$ Baris Kuskonmaz, ${ }^{2}$ Duygu Uckan, ${ }^{2}$ Sule Unal, ${ }^{2}$ Mualla Cetin ${ }^{2}$ and Fatma Gumruk ${ }^{2}$

${ }^{1}$ Hacettepe University Department of Pediatrics, and ${ }^{2}$ Hacettepe University Department of Pediatric Hematology, Ankara, Turkey

\section{ABSTRACT}

$\mathrm{F}$ amilial hemophagocytic lymphohistiocytosis is an autosomal reces$\checkmark$ sive, life-threatening condition characterized by defective immune response. A retrospective analysis was performed on 57 patients diagnosed with familial hemophagocytic lymphohistiocytosis at Hacettepe University Pediatric Hematology Department, Ankara, Turkey. Mutation analysis was performed on 37 patients, and of these: 11 had UNC13D, 10 had PRF1 and 3 had STX11 gene mutation. Of these patients, $44 \%$ were found to have central nervous system involvement on admission and spinal cord involvement was also seen in 5 patients. Remission was achieved in 24 patients with the treatment, in a median time of 76 days (min-max: 15-705 days). Time to remission was prolonged 3.1 times in patients with a ferritin level $1500 \mathrm{mg} / \mathrm{dL}$ or more. When patients were grouped according to age [Group 1 ( $\leq 2$ years), Group 2 (>2 years)]; patients in Group 1 had higher ferritin and aspartate aminotransferase levels but lower fibrinogen levels. The 5-year survival rate was also lower in Group 1. When patients in Group 1 were divided into two sub-groups according to hepatic involvement, the 5-year survival rate of patients who had hepatic involvement was significantly lower than those patients without hepatic involvement $(0.7 \%, 27 \%$, respectively) $(P=0.002)$. The 5 -year survival rate of patients who underwent hematopoietic stem cell transplantation was significantly higher than the patients who didn't $(44 \%, 16 \%$, respectively) $(P=0.02)$. In conclusion, age two years and under, ferritin level above $1500 \mathrm{mg} / \mathrm{dL}$, spinal cord or hepatic involvement should be considered as poor prognostic factors in familial hemophagocytic lymphohistiocytosis.

\section{Introduction}

Familial hemophagocytic lymphohistiocytosis (FHL) is a rare, autosomal recessive disease characterized by uncontrolled activation of $\mathrm{T}$ cells and macrophages, and excessive production of cytokines. ${ }^{1,2}$ The incidence of FHL in children varies in different studies. In Sweden, for example, it has been reported to be 1.2/1,000,000 while the frequency among hospitalized patients reported from Turkey is $1 / 1418{ }^{3}$ Although approximately $70 \%$ of patients with FHL are diagnosed before the age of one year, familial forms that manifest in late adulthood have also been reported. ${ }^{4,5}$ FHL is a genetically heterogeneous disorder and genotype-phenotype correlation is limited. So far, four genes (PRF1, UNC13D, STX11 and STXBP2) associated with FHL have been identified; unfortunately, in $10-50 \%$ of patients, none of these molecular defects can be determined. ${ }^{1,2}$

The most common symptoms of FHL are fever, hepatosplenomegaly and cytopenia. However, all organ systems can be involved at the time of diagnosis or during follow up. Jaundice, elevated liver enzymes, hepatomegaly, and coagulopathy are the signs of hepatobiliary involvement. ${ }^{6}$ Central nervous system (CNS) involvement has been reported to be associated with poor outcome and isolated CNS involvement can also be seen..$^{7-10}$

In this study, we aimed to evaluate the demographic, clinical and laboratory findings of FHL patients followed in Hacettepe University Children's Hospital and to
Haematologica 2018

Volume 103(2):231-236

\section{Correspondence:}

burcinbeken@gmail.com

Received: August 6, 2017.

Accepted: November 13, 2017.

Pre-published: November 16, 2017.

doi:10.3324/haematol.2017.178038

Check the online version for the most updated information on this article, online supplements, and information on authorship \& disclosures: www.haematologica.org/content/103/2/231

(C)2018 Ferrata Storti Foundation

Material published in Haematologica is covered by copyright. All rights are reserved to the Ferrata Storti Foundation. Use of published material is allowed under the following terms and conditions:

https://creativecommons.org/licenses/by-nc/4.0/legalcode. Copies of published material are allowed for personal or internal use. Sharing published material for non-commercial purposes is subject to the following conditions: https://creativecommons.org/licenses/by-nc/4.0/legalcode, sect. 3. Reproducing and sharing published material for commercial purposes is not allowed without permission in writing from the publisher. 
determine the risk factors affecting the treatment response, prognosis, and survival.

\section{Methods}

\section{Patients}

Retrospective, single center data were obtained from hemophagocytic lymphohistiocytosis (HLH) patients who were diagnosed between November 1994 and December 2012 in the Pediatric Hematology Department of Hacettepe University Children's Hospital, Ankara, Turkey. The HLH-94 and HLH-2004 diagnostic criteria of the Histiocyte Society were applied to confirm the diagnosis. ${ }^{11,12}$ Written informed consent was provided by the patients' parents and the study was approved by Hacettepe University Ethical Committee.

\section{Materials}

A diagnosis of FHL was made when there were affected siblings/close relatives (family history) or a history of consanguineous marriage, relapse, or when the molecular study was consistent with FHL. Patients with an underlying malignancy, metabolic disorder or rheumatological disease were excluded. The medical records of all patients were evaluated for demographic features, clinical and laboratory findings, bone marrow aspiration evaluation, mutation analysis and treatment received including hematopoietic stem cell transplantation (HSCT), complications, and mortality. CNS disease was evaluated by neurological findings such as neck stiffness, a bulging fontanelle, seizure, cranial nerve paralysis, hemiparesis, ataxia, drowsiness, and coma. Computed tomography (CT) or magnetic resonance imaging (MRI) findings indicative of involvement, or cerebrospinal fluid (CSF) protein level more than $30 \mathrm{mg} / \mathrm{dL}$ and/or a cell count of more than $50 \times 10^{6} / \mathrm{L}$ observed in the CSF (more than 5 white blood cells $/ \mathrm{mL}$ ) and/or evidence of hemophagocytosis in the CSF. ${ }^{13}$ Hepatic involvement was defined as elevated bilirubin, alanine and aspar- tate aminotransferase (ALT, AST) levels, low albumin levels, ascites and coagulopathy according to normal values by age. Complete remission and reactivation were decided on the basis of the criteria of the HLH society protocol. ${ }^{11}$

\section{Molecular genetic study for FHL}

A molecular genetic study was performed on the DNA samples of 37 patients by direct sequencing of all the coding exons as previously described in the literature. ${ }^{14}$

\section{Statistical analysis}

The software package SPSS v.16.0 was used in the statistical analysis of the data. Numerical variables were shown using mean \pm Standard Deviation (SD) and median (distribution) and qualitative variables were shown using numbers and percentages. The normality of the numerical variables was evaluated using the Shapiro Wilks test. To determine whether there was a difference with regard to qualitative variables between groups, the $\mathrm{X}^{2}$ test was used. To determine whether there was a difference with regard to numerical variables between two groups, the MannWhitney U test was used. Survival analyses were carried out using the Kaplan-Meier product-limit estimator method. Survival curves were compared using the log-rank test. To determine whether there was a difference with regard to numerical variables between more than two groups, the Kruskal-Wallis test was used. In determining the factors affecting survival, Cox regression analysis was used; $P<0.05$ was considered statistically significant.

\section{Results}

\section{Patients' characteristics}

Patients' characteristics and outcomes are shown in Table 1. A total of 37 patients were evaluated for mutations associated with FHL. It was determined that there were perforin mutations in a total of 10 patients, 4 of

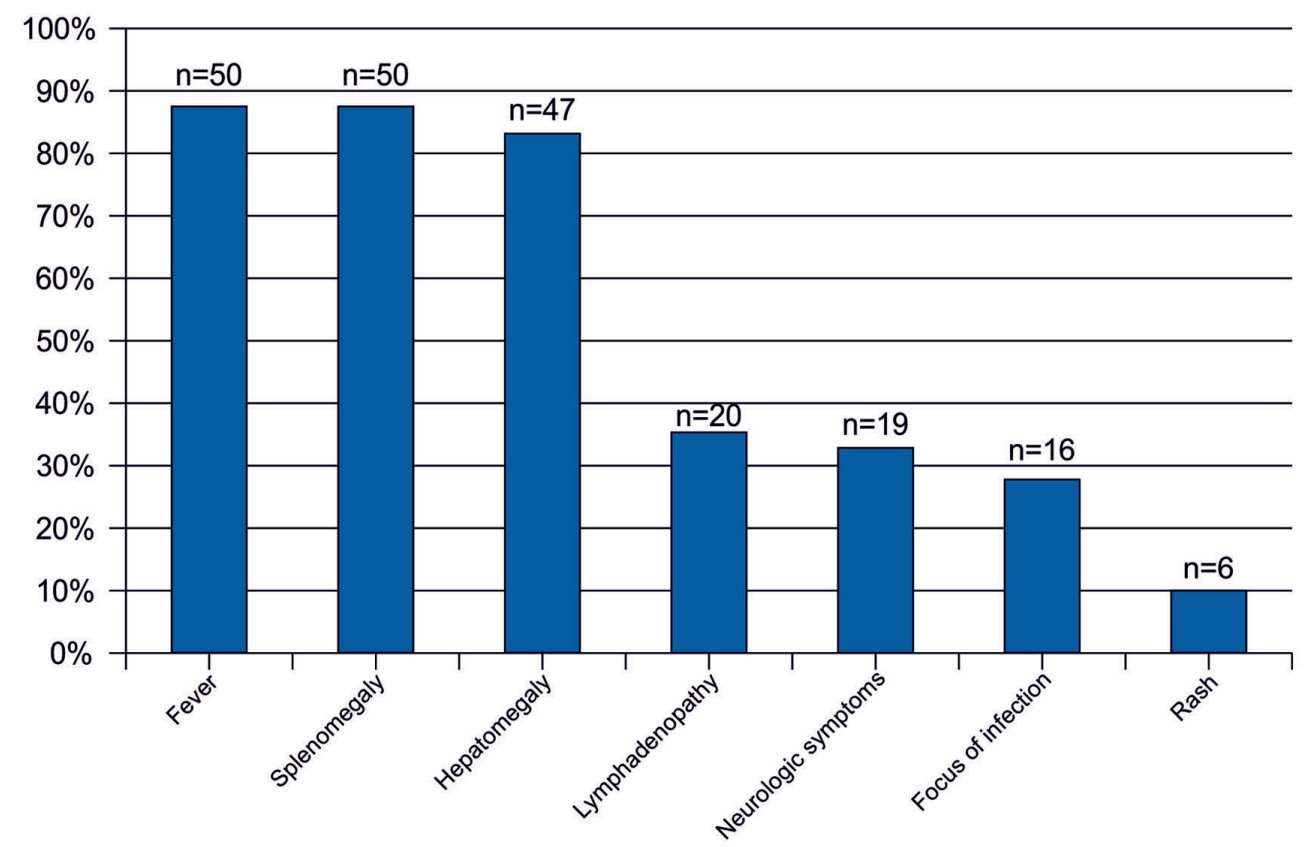

Figure 1. Clinical findings of patients at the time of diagnosis. Fever and hepatosplenomegaly were the most common symptoms at the time of diagnosis. 
whom had been previously published. ${ }^{14,15}$ UNC13D and STX11 mutations were detected in 11 and 3 patients, respectively. There was no statistical difference in terms of demographic, clinical and laboratory findings, and survival between the mutation positive and negative groups. A history of a deceased sibling was significantly higher in mutation-positive patients $(P=0.027)$. Serological evaluation for the Epstein-Barr virus (EBV) was performed in 41 patients and was compatible with previous EBV infection in 32 patients $(78 \%)$ and active infection in one patient $(2 \%)$. The patient was also treated with intravenous ganciclovir. Clinical features of the patients are shown in Figure 1.

\section{Comparison of clinical and laboratory findings according to the patients' initial age at diagnosis}

Patients were categorized into two groups according to age: $\leq 2$ years (Group 1) and $>2$ years (Group 2). The comparison of the laboratory findings and the 5-year survival rates of these two groups are shown in Table 2 .

\section{Comparison of clinical and laboratory findings according to hepatic involvement}

Twenty-six (46\%) patients had hepatic involvement at the time of diagnosis; $15(58 \%)$ of these patients were two years old or younger, and the remaining $11(42 \%)$ were older than two years of age when they were diagnosed. There was no statistical difference in 5-year survival rates between patients with hepatic involvement and without hepatic involvement $(20 \%, 26 \%$, respectively) $(P=0.210)$. Analysis of the patients under two years old for hepatic involvement revealed that the 5-year survival rate of the patients with hepatic involvement was significantly lower than those without hepatic involvement $(0.7 \%, 27 \%$, respectively) $(P=0.002)$.

\section{Comparison of clinical and laboratory findings according to CNS involvement}

Twenty-five patients (44\%) had CNS involvement at the time of diagnosis and 17 patients $(30 \%)$ developed CNS involvement during follow up. While convulsion was the most common (48\%) neurological symptom, the others reported were: cranial nerve involvement, hemiparesis, somnolence, confusion, headache, neck stiffness, delirium and ataxia. Five patients did not have neurological symptoms, but either MRI or CSF findings (elevated protein, histiocytes causing moderate pleocytosis, or hemophagocytosis) were compatible with HLH involvement.

There were 6 patients diagnosed over ten years of age; 3 of these patients (10,14 and 15 years old) had isolated CNS involvement at the time of diagnosis and all died despite undergoing HSCT. Two patients had both CNS and bone marrow infiltration (one of them is still alive) and one patient had only bone marrow infiltration (still alive).

Spinal cord involvement was determined in 5 patients, including one at diagnosis and 4 during follow up; one of these patients has been previously published..$^{16}$ Median age of the patients with spinal cord involvement was 11 years and all had neurological symptoms at the time of diagnosis. All of the patients with spinal cord involvement died despite 4 undergoing HSCT. Three patients had isolated CNS involvement at the time of diagnosis and 2 of them had concomitant spinal cord involvement. The clinical features of patients with spinal cord involvement are shown in Online Supplementary Table S1.

The female/male ratio of patients with CNS involvement at the time of diagnosis was significantly higher than those without (3.1/1, 0.6/1, respectively) $(P=0.015)$. Median age at diagnosis was higher in patients with CNS involvement than those without (36 months, 10.5 months, respectively) $(P=0.028)$. There was no difference in 5 -year survival rates between the two groups.

\section{Comparison of HLH-94 and HLH-2004 treatment protocols, HSCT results and outcomes}

Thirty-two patients (56\%) were treated according to the HLH-2004 protocol, 17 patients $(30 \%)$ were treated according to the HLH-1994 protocol, and 8 patients $(14 \%)$ received only supportive care (those patients in a poor clinical condition and who died before initiation of treatment). There were no statistical differences in remission

Table 1. Patients' characteristics, some clinical findings and outcomes.

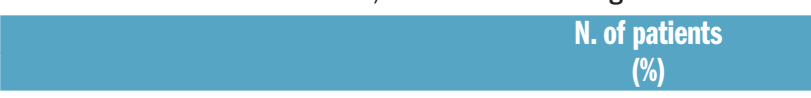

Sex

$\begin{array}{ll}\text { Female } & 32(56 \%) \\ \text { Male } & 25(44 \%)\end{array}$

Consanguinity between parents

$40(70 \%)$

$(-)$ $17(30 \%)$

History of an affected sibling

CNS involvement on admission

$\begin{array}{lc}(+) & 25(44 \%) \\ (-) & 32(56 \%) \\ \text { Molecular genetic analysis } & 37(65 \%) \\ \text { UNC13D } & 11(30 \%) \\ \text { PRF1 } & 10(27 \%) \\ \text { STX11 } & 3(8 \%) \\ \text { Undetectable } & 13(35 \%)\end{array}$

Treatment protocol

HLH-1994

$17(30 \%)$

HLH-2004

$32(56 \%)$

Supportive treatment $8(14 \%)$

HSCT

$(+)$

$18(32 \%)$

$(-)$

$39(68 \%)$

Relapse

$20(35 \%)$

Bone marrow

CNS

Bone marrow and CNS

$14(70 \%)$

\section{Outcome}

Alive without disease

11(19\%)

Dead

Patients with HSCT: 9

Patients without HSCT: 2

Patients with HSCT: 9

46(81\%)

Patients without HSCT: 37

N.: number; CNS: central nervous system; HSCT: hematopoietic stem cell transplantation. 
(35\%, 56\%, respectively) ( $P=0.27)$ and 5 -year survival rates $(37 \%, 17 \%$, respectively) $(P=0.17)$ between HLH1994 and HLH-2004 treatment protocols.

Eighteen patients $(32 \%)$ underwent HSCT. Nine were lost because of subsequent complications. The 5 -year survival rate of patients with HSCT were significantly higher than those without HSCT (44\%, 16\%, respectively)
$(P=0.02)$. Twenty-four patients went into remission with a median time of 76 days (15-705 days). Among the factors that could affect the remission time, a ferritin level higher than $1500 \mathrm{ng} / \mathrm{mL}$ was found to extend the time to remission by 2.3 times (95\% CI: 0.9-5.7). When data were evaluated through multiple-variable analysis with triglycerides, fibrinogen, and sodium levels, a ferritin level more

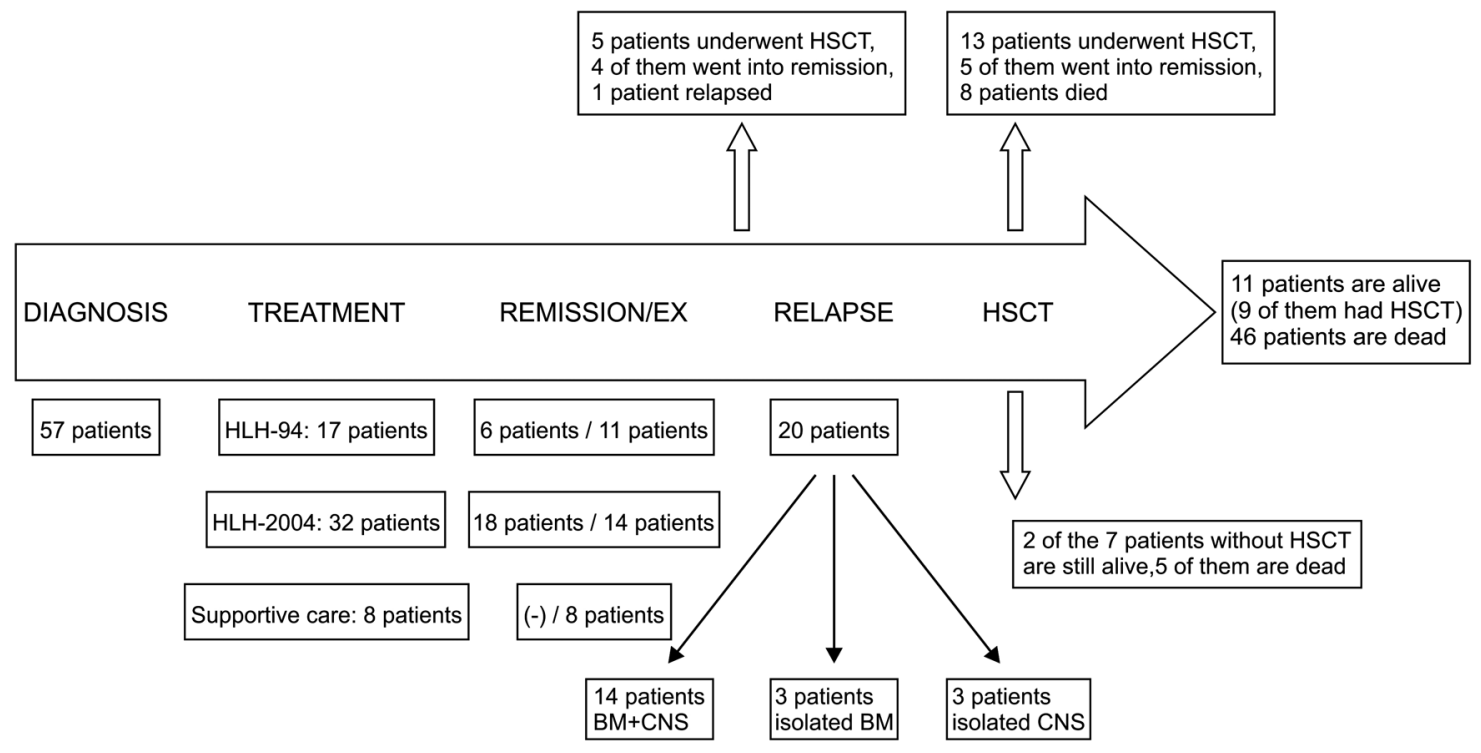

Figure 2. Patient follow-up shown as a flow chart. EX: Exitus; BM: bone marrow; CNS: central nerveous system; HLH: hemophagocytic lymphohistiocytosis; HSCT: hematopoetic stem cell transplantation.

Table 2. Comparison of laboratory findings and survival according to age at diagnosis.

\begin{tabular}{|c|c|c|c|}
\hline Laboratory findings & $\begin{array}{c}\text { Group } 1 \\
\text { ( } \leq 2 \text { years old) } \\
n=33 \\
\text { Median (min-max) }\end{array}$ & $\begin{array}{c}\text { Group } 2 \\
(>2 \text { years old) } \\
n=24 \\
\text { Median (min-max) }\end{array}$ & $P$ \\
\hline Hemoglobin $(\mathrm{g} / \mathrm{dL})$ & $7.9(3.6-11.8)$ & $8.3(4.9-13.1)$ & 0.682 \\
\hline Leukocyte $\left(\times 10^{9} / \mathrm{L}\right)$ & $6.1(0.8-16.5)$ & $4.3(0.4-10.7)$ & 0.672 \\
\hline Thrombocyte $\left(\times 10^{9} / \mathrm{L}\right)$ & $29(7-683)$ & $41(4-177)$ & 0.477 \\
\hline Ferritin (ng/dL) & $1963(128-51788)$ & $1104(133-7718)$ & 0.001 \\
\hline Triglyceride (mg/dL) & 347 (41-1443) & $358(43-872)$ & 0.959 \\
\hline Fibrinogen (mg/dL) & $123(10-624)$ & $223(2-483)$ & 0.023 \\
\hline $\operatorname{ALT}(\mathrm{U} / \mathrm{L})$ & $73(13-539)$ & $69(5-466)$ & 0.884 \\
\hline AST (U/L) & $104(16-1894)$ & $71(11-373)$ & 0.043 \\
\hline Total bilirubin (mg/dL) & $1.1(0.1-25.2)$ & $1.3(0.1-15.5)$ & 0.722 \\
\hline Conjugated bilirubin (mg/dL) & $0.6(0.1-17.4)$ & $0.6(0.1-11.6)$ & 0.686 \\
\hline $\mathrm{LDH}(\mathrm{U} / \mathrm{L})$ & $1080(258-2583)$ & $790(76-5730)$ & 0.156 \\
\hline Total protein (mg/dL) & $5.4(3.6-7.0)$ & $5.5(3.6-7.6)$ & 0.945 \\
\hline Albumin (g/dL) & $3.0(1.9-4.0)$ & $3.2(1.7-4.2)$ & 0.597 \\
\hline Sodium $(\mathrm{mmol} / \mathrm{L})$ & $134(125-150)$ & $136(120-145)$ & 0.841 \\
\hline aPTT (sn) & $37(22-180)$ & $33(24-66)$ & 0.057 \\
\hline 5-year survival rate & $19 \%$ & $33 \%$ & 0.017 \\
\hline
\end{tabular}

ALT: alanine aminotransferase; AST: aspartate aminotransferase; aPTT: activated partial thromboplastine time; LDH: lactate dehydrogenase. 
than $1500 \mathrm{ng} / \mathrm{mL}$ was found to extend the time to remission by 3.1 times (95\% CI: 1.1-8.5).

As for the factors that affect the 5-year survival rate of the patients who did not undergo HSCT, it was seen that sex, age of diagnosis, CNS involvement, and relapse did not have any effect on survival. When the data with $P$-values over 0.250 (aPTT, sodium, triglycerides, albumin and relapse) were analyzed with multivariate and Cox regression analysis, a low sodium level $(<135 \mathrm{mEq} / \mathrm{L})$ was found to increase the mortality risk by 2.46 times (95\% CI: 1.1 5.3). Follow up of the patients is given as a flowchart in Figure 2.

\section{Discussion}

Familial hemophagocytic lymphohistiocytosis is an autosomal recessive, life-threatening, inflammatory condition with excessive, prolonged, and ineffective immune response. It is expected to become frequent in Turkey because of the high rates of consanguineous marriages. ${ }^{3}$

Median age of our patients was 18 months and $47 \%$ of the patients were diagnosed before the age of one. According to the literature, $70 \%$ of FHL patients are diagnosed in the first year of life. The median age of diagnosis of our patients was thought to be higher because of the 6 patients over ten years of age in our study group. We think that the mean age of FHL diagnosis in the literature will increase with the growing number of adolescent and adult patients reported in recent years.

The most common clinical findings on admission were fever, splenomegaly, focus of infection, lymphadenopathy, and rash, and these are similar to the FHL international registry (Figure 3$)^{5}$

When we analyzed the mutations responsible for FHL, we found the rate of UNC13D mutation slightly higher than in the literature. ${ }^{17}$ The median ages of diagnosis were the same as in the literature: three months in PRF1 mutation and four months in UNC13D mutation. ${ }^{18}$ The median age at diagnosis was 89 months in STX11 mutation. A study by Rudd et al. found STX11 mutation in 6 of 34 patients, half of whom were diagnosed after one year of age and who, unexpectedly, were able to survive for a long period without treatment. ${ }^{19}$

The 5-year survival rate of the patients who were two years old or under at the time of diagnosis was observed to be significantly lower than the patients over two years of age. On the other hand, when these two groups were compared in terms of laboratory findings, it became apparent that the patients aged two years or under had lower fibrinogen levels, and higher ferritin and AST levels. These parameters, which are related to disease activation have also been observed as being associated with a worse prognosis in other studies. ${ }^{3,20}$ Taking into consideration the fact that the liver is the site of fibrinogen synthesis, low levels of fibrinogen and elevated AST levels are considered to be associated with liver involvement. We also found that, in the age two years or under patient group, those with hepatic involvement had lower survival rates. In 2009, Flipovich had suggested that liver involvement should be included in the diagnostic criteria. ${ }^{21}$ There is also a study reporting poor prognosis in cases of secondary $\mathrm{HLH}$ presenting with acute hepatic failure. ${ }^{22}$

In $44 \%$ of our patients, CNS involvement was detected at the time of diagnosis, and in $14 \%$ it was detected during follow up. According to the literature, $10-73 \%$ of patients with FHL have CNS involvement at the time of diagnosis. ${ }^{2,8,15,16}$ Haddad et al. reported that CNS involvement occurs in particular during the reactivation of the disease, and that all patients without HSCT develop CNS complications. In their study, 39\% of the patients had CNS involvement at the time of diagnosis, a percentage that increased to $54 \%$ when patients with relapse were included. ${ }^{8}$ A study by Horne et al. which focused on the frequency of CNS involvement in $193 \mathrm{HLH}$ patients and the characteristics of these patients, reported that patients with CNS involvement were diagnosed at a median of nine months and that $55 \%$ of these were under one year of age when diagnosed..$^{13}$

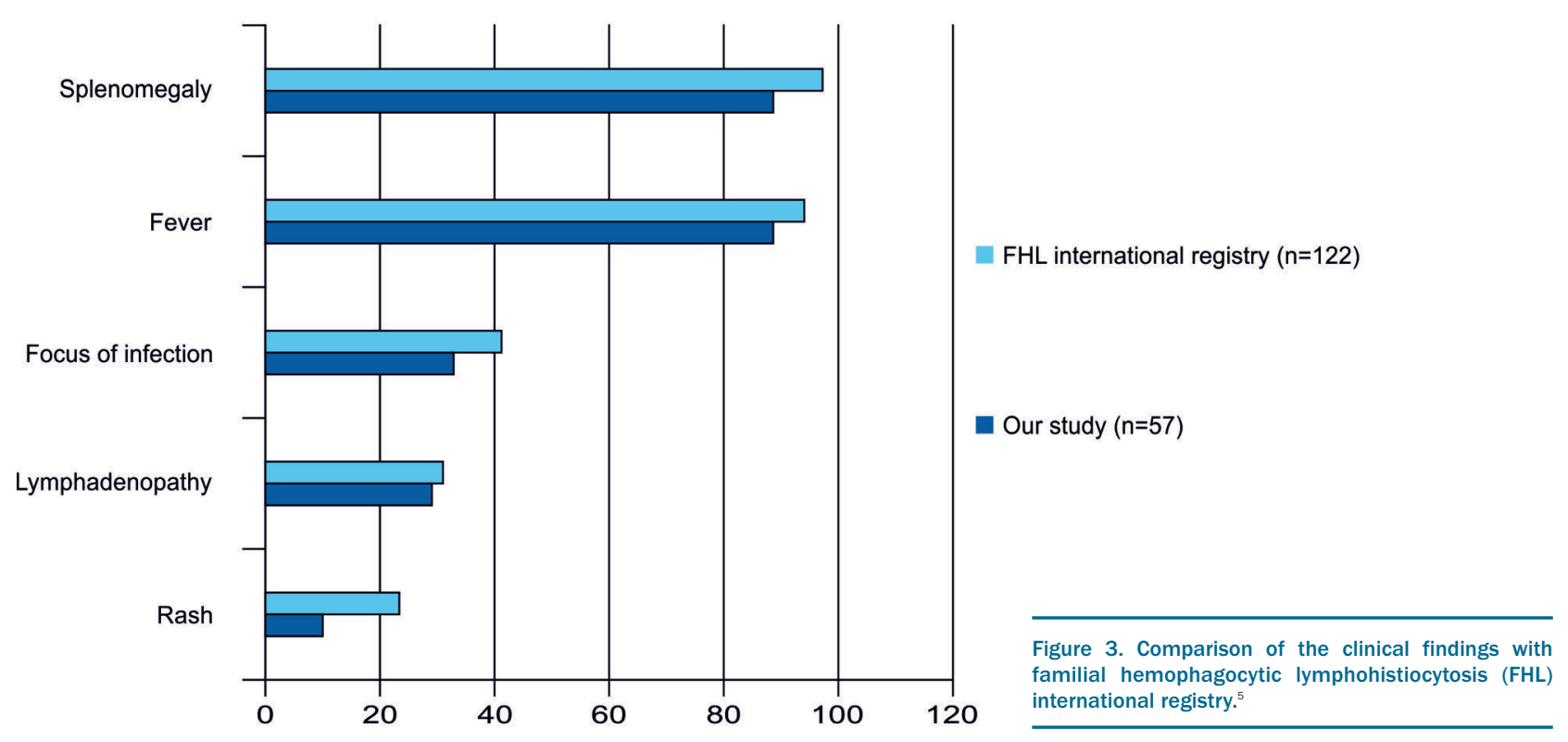


In our study, the median age of diagnosis in patients with CNS involvement was found to be 36 months.

Three of our patients had isolated CNS involvement at the time of diagnosis. Even though a few patients were reported to have isolated CNS involvement, we believe that the number of such cases is not limited to those who have been reported because it is rather difficult to diagnose patients with isolated CNS symptoms, which leads to misdiagnosis and/or mistreatment. ${ }^{10,23,24}$

Although spinal cord involvement in FHL is a rare entity, and thus only a few patients have been reported in the literature to date, ${ }^{9,16}$ it was detected in $9 \%$ of our patients. We suggest that a cranial MRI, accompanied by a spinal MRI should be carried out in patients older than ten years of age and who have neurological symptoms in order to verify whether they have spinal cord involvement or not. Although it is a well-known fact that CNS involvement has a negative effect on FHL prognosis, ${ }^{8,13,15}$ we did not find any differences between the survival rates of patients with and those without CNS involvement.

Approximately $33 \%$ of our patients underwent HSCT.
Their 5 -year survival rate was found to be $44 \%$, similar to that reported in the literature (49-66\%).

When the factors affecting remission time were analyzed, it was seen that ferritin level higher than 1500 $\mathrm{ng} / \mathrm{mL}$ extends the time to remission by 3.1 times. In 2012, Trottestam et al. demonstrated that elevated ferritin, bilirubin, creatinine, and aPTT levels together with low albumin levels, and the presence of pleocytosis in the CSF at the time of diagnosis, have an impact on early mortality, and a ferritin level more than $2000 \mathrm{ng} / \mathrm{mL}$ increased mortality by 3.2 times. $^{20}$ It can be assumed that high mortality rates in patients with elevated ferritin levels are related to poor response to treatment.

In conclusion, FHL is a disease with a fatal course, its only curative treatment being HSCT. Mutation analysis should be performed immediately in HLH patients in order to avoid any delay for HSCT. Patients aged two years and under, those with a ferritin level more than 1500 $\mathrm{mg} / \mathrm{dL}$ at the time of diagnosis, and who have spinal cord or hepatic involvement, should be considered as having a poor prognosis.

\section{References}

1. Gholam C, Grigoriadou S, Gilmour KC, Gaspar HB. Familial haemophagocytic lymphohistiocytosis: advances in the genetic basis, diagnosis and management. Clin Exp Immunol. 2011;163(3):271-283

2. Janka GE. Familial and Acquired Hemophagocytic Lymphohistiocytosis. Annu Rev Med. 2012;63:233-246.

3. Gurgey A, Gogus S, Ozyurek E, et al. Primary hemophagocytic lymphohistiocytosis in Turkish children. Pediatr Hematol Oncol. 2003;20(5):367-371.

4. Henter JI, Elinder G, Söder O, Ost A. Incidence in Sweden and clinical features of familial hemophagocytic lymphohistiocytosis. Acta Paediatr Scand. 1991; 80(4):428435.

5. Aricò M, Janka G, Fischer A, et al. Hemophagocytic lymphohistiocytosis. Report of 122 children from the International Registry. FHL Study Group of the Histiocyte Society. Leukemia. 1996;10(2):197-203.

6. Guthery SL, Heubi JE. Liver involvement in childhood histiocytic syndromes. Curr Opin Gastroenterol. 2001;17(5):474-478.

7. Henter JI, Nennesmo I. Neuropathologic findings and neurologic symptoms in twenty-three children with hemophagocytic lymphohistiocytosis. J Pediatr. 1997;130(3):358-365.

8. Haddad E, Sulis ML, Jabado N, Blanche S, Fischer A, Tardieu M. Frequency and severity of central nervous system lesions in hemophagocytic lymphohistiocytosis. Blood. 1997;89(3):794-800.

9. Chiapparini L, Uziel G, Vallinoto C, et al. Hemophagocytic lymphohistiocytosis with neurological presentation: MRI findings and a nearly miss diagnosis. Neurol Sci. 2011;32(3):473-477.
10. Rostasy K, Kolb R, Pohl D, et al. Central nervous system disease as the main manifestation of hemophagocytic lymphohistiocytosis in two cildren. Neuropediatrics. 2004;35(1):45-49.

11. Henter JI, Horne A, Aricó M, et al. HLH2004: Diagnostic and Therapeutic Guidelines for Hemophagocytic Lymphohistiocytosis. Pediatr Blood Cancer. 2007;48(2):124-131.

12. Henter J-I, Arico M, Egeler M, et al. HLH-94 A treatment protocol for hemophagocytic lymphohistiocytosis. Med Pediatr Oncol. 1997;28(5):342-347

13. Horne A, Trottestam H, Arico M, et al. Frequency and spectrum of central nervous system involvement in 193 children with haemophagocytic lymphohistiocytosis. $\mathrm{Br} \mathrm{J}$ Haematol. 2008;140(3):327-335.

14. Okur H, Balta G, Akarsu N, et al. Clinical and molecular aspects of Turkish familial hemophagocytic lymphohistiocytosis patients with Perforin mutations. Leuk Res. 2008;32(6):972-975

15. Gurgey A, Aytac S, Balta G, Oguz KK Gumruk F. Central nervous system involvment in Turkish children with primary hemophagocytic lymphohistiocytosis. J Child Neurol. 2008;23(11):1293-1299.

16. Gokce M, Balta G, Unal S, Oguz KK, Cetin $M$, Gumruk F. Spinal cord involvement in a child with familial hemophagocytic lymphohistiocytosis. J Pediatr Neurosci. 2012;7(3):194-196.

17. Zur Stadt U, Beutel K, Kolberg S, et al. Mutation spectrum in children with primary hemophagocytic lymphohistiocytosis: molecular and functional analyses of PRF1, UNC13D, STX11, and RAB27A. Hum Mutat. 2006;27(1):62-68.

18. Sieni E, Cetica V, Santoro A, et al. Genotype-phenotype study of familial haemophagocytic lymphohistiocytosis type
3. J Med Genet. 2011:48(5):343-352

19. Rudd E, Göransdotter Ericson K, Zheng C et al. Spectrum and clinical implications of syntaxin 11 gene mutations in familial haemophagocytic lymphohistiocytosis: association with disease-free remissions and haematopoietic malignancies. J Med Genet. 2006;43(4):e14

20. Trottestam H, Berglöf E, Horne A, et al. Risk factors for early death in children with haemophagocytic lymphohistiocytosis. Acta Paediatr. 2012;101(3):313-318.

21. Filipovich $\mathrm{AH}$. The expanding spectrum of hemophagocytic lymphohistiocytosis. Curr Opin Allergy Clin Immunol. 2011; 11(6):512-516.

22. leri T, Azık F, Uysal Z, Ertem M, Kulo lu Z, Gözda o lu S. Severe acute hepatic failure as an initial manifestation of hemophagocyticlymphohistiocytosis. Ankara Universites Tip Fakültesi Mecmuasi. 2009; 62:119-123.

23. Rooms L, Fitzgerald N, McClain K.L. Hemophagocytic lymphohistiocytosis masquerading as child abuse: presentation of three cases and review of central nervous system findings in hemophagocytic lymphohistiocytosis. Pediatrics. 2003;111(5 Pt 1):e636-640.

24. Trutzo LC, Lin DD, Hartung H, et al. A neurologic presentation of familial hemophagocytic lymphohistiocytosis which mimicked septic emboli to the brain. J Child Neurol. 2007;22(7):863-868.

25. Ouachée-Chardin M, Elie C, de Saint Basile $G$, et al. Hematopoietic stem cell transplantation in hemophagocytic lymphohistiocytosis: a single-center report of 48 patients. Pediatrics. 2006;117(4):e743-750.

26. Yoon HS, Im HJ, Moon HN, et al. The outcome of hematopoietic stem cell transplantation in Korean children with hemophagocytic lymphohistiocytosis. Pediatr Transplant. 2010;14(6):735-740. 